\title{
Granuloma anulare giganteum et disseminatum
}

\section{Granuloma annulare giganteum et disseminatum}

Autoren

Institut
U. Proske, G. Wozel

Klinik und Poliklinik für Dermatologie, Universitätsklinikum Carl Gustav Carus, Technische Universität Dresden

\section{Bibliografie}

DOI http://dx.doi.org/

10.1055/s-0030-1256459

Akt Dermatol 2011; 37:

210-213 @ Georg Thieme

Verlag KG Stuttgart · New York

ISSN 0340-2541

Korrespondenzadresse

ö̈ Dr. med. Ulrike Proske

Klinik und Poliklinik

für Dermatologie

Universitätsklinikum

Carl Gustav Carus

TU Dresden

Fetscherstraße 74

01307 Dresden

Ulrike.Proske@

uniklinikum-dresden.de

\section{Zusammenfassung \\ $\nabla$}

Das Granuloma anulare ist eine nicht infektiöse Erkrankung unklarer Ätiologie. Es ist gekennzeichnet durch eine granulomatöse Entzündungsreaktion. Neben lokalisierten, oft selbst limitiert verlaufenden Formen sind disseminierte Verläufe möglich. Eine seltene Manifestationsform ist ein Granuloma anulare giganteum. Darunter versteht man eine plaqueförmige Läsion mit einer Größenausdehnung bis zu mehreren Zentimetern. Wir berichten über einen Patienten mit einem Granuloma anulare giganteum et disseminatum, der während der Behandlung eine Hyperthyreose entwickelte. Trotz lokaler Therapie mit Glukokortikosteroiden und mehrmaliger Injektionen einer Steroid-Kristallsuspension so-

\section{Einleitung}

Das Granuloma anulare ist eine benigne entzündliche Hauterkrankung, die durch gruppierte oder in Ringen angeordnete Papeln und histomorphologisch nachweisbare Granulome gekennzeichnet ist. Die auslösende Ursache ist beim Granuloma anulare weiterhin unklar; Assoziationen mit Diabetes mellitus Typ 1, Arthritiden und Schilddrüsenerkrankungen, speziell Autoimmunthyreoiditiden bei Frauen, sind beschrieben [1]. Auch Zusammenhänge mit Arthritiden sind bekannt [2]. Bevorzugte Lokalisationen sind die Akren, besonders die Dorsalseite von Händen, Fingern, Füßen und Zehen sowie Ellenbogen oder Knie. Es handelt sich primär um gerötete oder hautfarbene Papeln, die sich nach peripher ausbreiten, wobei das Zentrum später in das Hautniveau zurücksinkt. Dadurch entstehen die typischen ringförmigen Herde, die mehrere Zentimeter Größe und bizarre Konfigurationen erreichen können. Man unterscheidet verschiedene Sonderformen wie die subkutane Form mit hautfarbenen derben wie der internen Therapie mit einem Antimalariamittel kam es zu weiterer Progression der Erkrankung. Nach Behandlungsbeginn mit Fumarsäureester (FSE) in dem für die Psoriasis empfohlenen Dosierungsschema zeigte sich eine langsame Regredienz. Allerdings berichtete der Patient ab einer Dosierung von 4 Tabletten Fumaderm ${ }^{\circledR}$ pro Tag über Übelkeit und Durchfälle. Die Diagnose der Hyperthyreose wurde verzögert gestellt, da die beschriebenen Beschwerden zunächst als unerwünschte Wirkungen der Therapie mit FSE gewertet wurden. Es sollte deshalb bei Zunahme von Durchfällen, rascher Gewichtsabnahme, Unruhe und Tachykardie an die Entwicklung einer Hyperthyreose gedacht und entsprechende Laborwerte kontrolliert werden.

\section{Fallbericht $\nabla$}

\section{Anamnese}

Ein 44-jähriger Patient wurde erstmals im Oktober 2005 in unserer Klinik mit einem histologisch gesicherten Granuloma anulare vorgestellt. Im Juni 2005 war ihm eine ringförmige papulöse Hautveränderung im Bereich des Nackens aufgefallen, die langsam weiter an Größe zugenommen hat. Vorerkrankungen bestanden nicht. Ein Diabetes mellitus und eine Schilddrüsenerkrankung wurden vor Behandlungsbeginn ausgeschlossen. Größe $173 \mathrm{~cm}$, Gewicht $76 \mathrm{~kg}$ bei Behandlungsbeginn, $73 \mathrm{~kg}$ bei Behandlungsende. 


\section{Erstbefund}

Bei der Erstvorstellung zeigte sich am Hals dorsal eine ovaläre gerötete, 3,5 × 2,5 cm große, kombinierte Effloreszenz aus zentral eingesunkener Makula und erhabenem Randwall ( $\bullet$ Abb. 1).

\section{Diagnostik}

Laborbefunde: NBZ, Blutbild, NA, K, CREA, ALAT, ASAT, GGT, AP, TSH, MAK, TAK, TRAK, Quick, INR im Normbereich.

Mykologische Diagnostik: Nachweis von Pilzen im Nativpräparat, Kultur negativ.

Mikrobiologische Diagnostik: Im Abstrich Keime nicht nachgewiesen, Kultur aerob steril.

Histologie: Deutliche granulomatöse Entzündung mit Nachweis von histiozytären Palisadengranulomen und zentralen nekrobiotischen Kollagenfaserdegenerationen mit Muzinnachweis. In der Pilz-PAS-Spezialfärbung eindeutiger Nachweis von Pilzelementen in den Follikelostien.

\section{Therapie und Verlauf}

Wegen des Nachweises von Pilzelementen im Bioptat und im Nativpräparat wurde eine interne Therapie mit Terbinafin über insgesamt 8 Wochen eingeleitet. Darunter anfänglich leichte Regredienz der Veränderung, dann jedoch weitere Progression. Dazu kamen im Verlauf des folgenden Jahres weitere Herde an der linken Ohrmuschel, am 2. Fingerzwischenraum links und am rechten Oberarm. Der Herd am Hals zeigte eine Größenzunahme auf $9 \times 9 \mathrm{~cm}(\circlearrowleft$ Abb. 2a,b). Die Behandlung war zwischenzeitlich mit lokalen Glukokortikosteroiden (Clobetasol-Creme) und intraläsionalen Glukokortikosteroid-Injektionen erfolgt. Es zeigte sich jedoch eine weitere Größenzunahme des Herdes am Hals auf 12 $\times 11 \mathrm{~cm}$ mit Bewegungseinschränkung ( $\bullet$ Abb. 3a,b). Auch während der internen Behandlung mit Hydroxychloroquin ab Dezember 2006 ( $400 \mathrm{mg} / \mathrm{d}$, entspricht 5,2 mg/kg KG) weitere Progression der Hautveränderung am Nacken und Auftreten eines neuen Plaque am linken Oberarm. Daraufhin Beendigung der Hydroxychloroquin-Therapie im März 2007 und Einstellung auf FSE (Beginn mit Fumaderm initial ${ }^{\circledR}$ in Analogie zur Standardtherapie bei Psoriasis). Nach 12 Wochen Therapie bei einer Dosis von 5 Tabletten Fumaderm ${ }^{\circledR}$ pro Tag war ein Sistieren der Größenzunahme und eine langsame Abflachung der Granulome zu verzeichnen. Ab einer Dosis von 4 Tabletten Fumaderm ${ }^{\circledR}$ pro Tag klagte der Patient zunehmend über Magenbeschwerden und Durchfälle. Zusätzlich zeigte sich eine Lymphopenie $(0,57 \mathrm{Gpt} / \mathrm{l})$. Unter langsamer Dosisreduktion konnte die Medikation nach 6 Monaten Behandlung bei sichtbarer Verkleinerung und Abflachung der Hautveränderungen abgesetzt werden. Nach weiteren 2 Monaten bestand im Nackenbereich eine Narbe, die anderen betroffenen Areale waren narbenlos, partiell mit Hyperpigmentierung, abgeheilt $(\boldsymbol{\otimes} \mathbf{A b b} . \mathbf{4 a}, \mathbf{b})$.

Im Rahmen der Blutkontrolle zeigte sich 6 Monate nach Therapiebeginn eine Hyperthyreose. Die zunehmenden Durchfälle sowie ein Gewichtsverlust von $3 \mathrm{~kg}$ innerhalb der letzten 6 Wochen waren wegweisend für die Diagnosestellung. Die Behandlung erfolgte mit Methimazol und später einer Radiojodtherapie. Ein Zusammenhang mit der Medikation mit FSE wurde nicht gesehen.

\section{Diskussion}

Das Granuloma anulare gehört zu den granulomatösen Dermatosen, wobei die Ätiologie weitgehend unklar ist. Als Triggerfaktoren wurden Insektenstiche und andere Traumata, Impfungen,

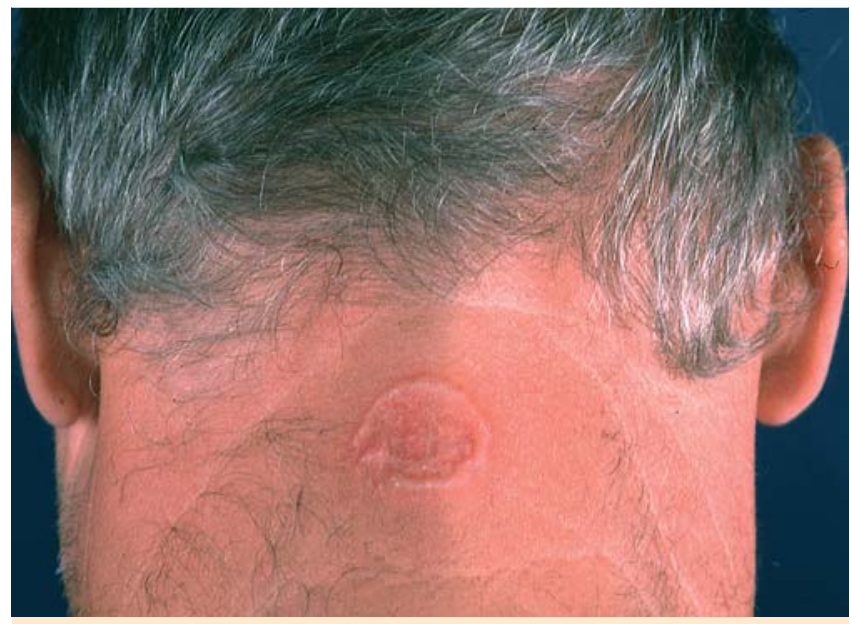

Abb. 1 Hautbefund am Hals 21. 10. 2005.
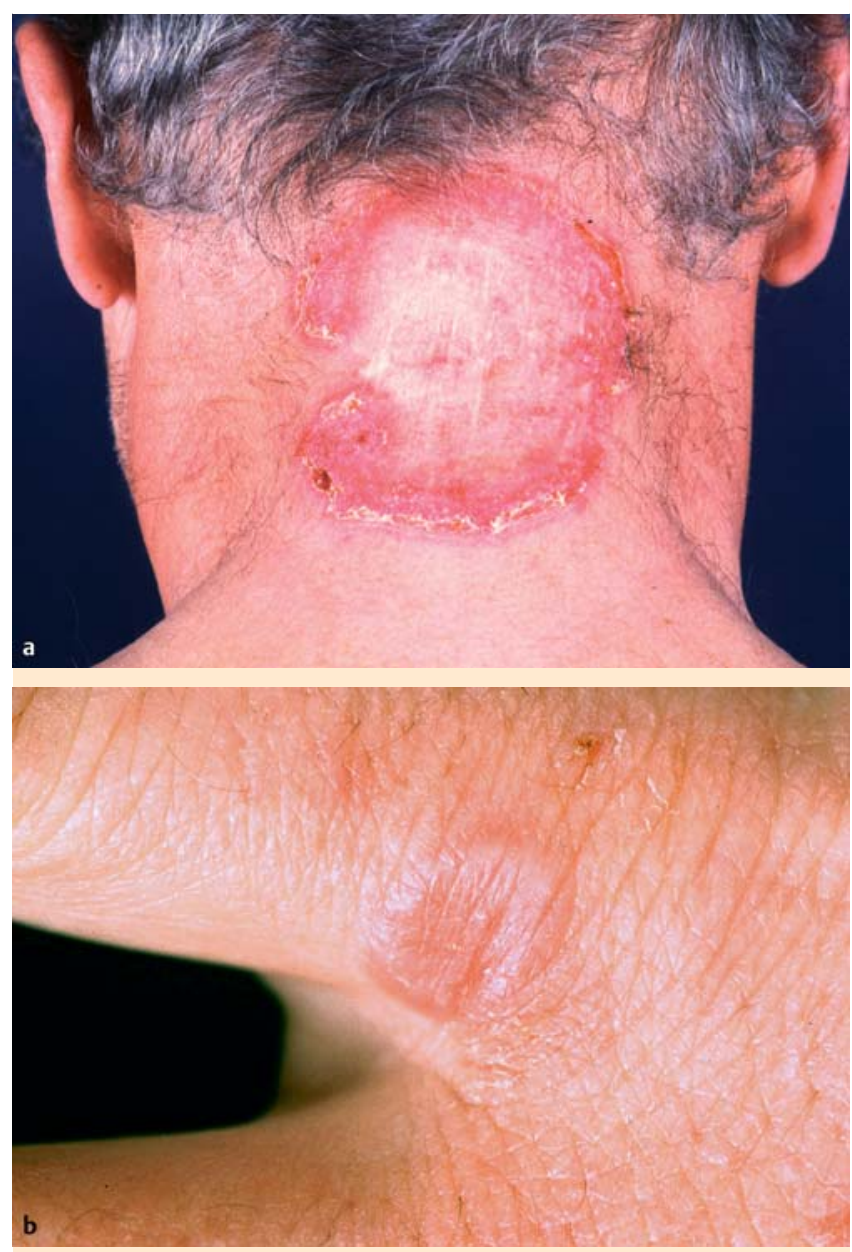

Abb. 2 Hautbefund am Hals (a) und Handrücken (b) 17. 01. 2007.

Sonnenexposition, virale Infekte oder persistierende mechanische Reize diskutiert. Assoziationen mit Diabetes mellitus Typ 1 und Autoimmunthyreoiditiden sind beschrieben $[1,5]$.

Die granulomatösen Infiltrate werden möglicherweise durch zirkulierende Immunkomplexe und die Ausbildung einer Immunkomplexvaskulitis verursacht [5,6]. Hinweise auf eine lokale Aktivierung der spezifischen zellulären Immunität ergeben sich aus dem Nachweis von aktivierten CD4+-Lymphozyten im entzündlichen Infiltrat [1]. Der Nachweis aktivierter Lymphozyten, vor- 

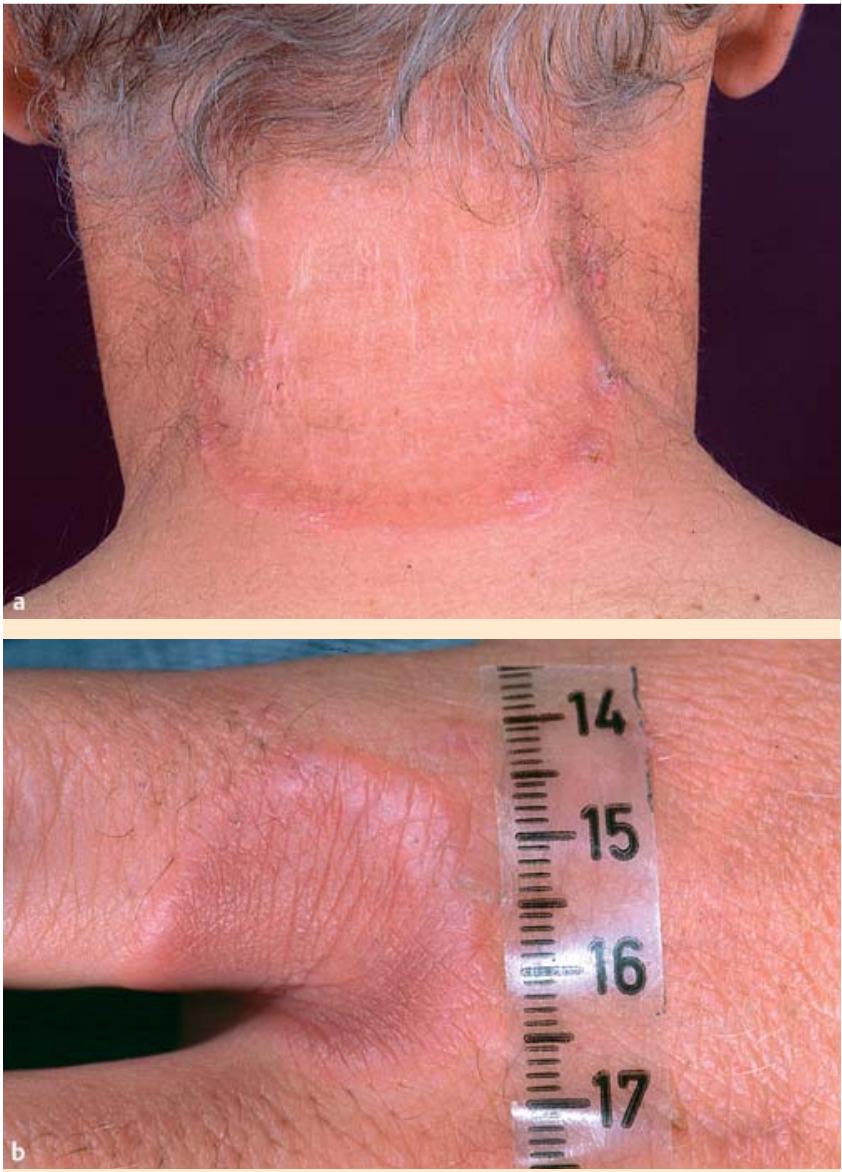

Abb. 3 Hautbefund am Hals (a) und Handrücken (b) 13. 08. 2007.

nehmlich T-Helferzellen, unterstützt die Hypothese einer zellvermittelten Immunreaktion vom Spättyp auf ein unbekanntes Antigen [7].

Zur Behandlung des Granuloma anulare stehen verschiedene therapeutische Optionen mit unterschiedlichen Erfolgen zur Verfügung. Für das lokalisierte Granuloma anulare mit hoher Spontanheilungstendenz ist therapeutische Zurückhaltung gerechtfertigt [8].

Für die Lokaltherapie sind Glukokortikosteroide, ggf. unter Okklusivverband oder als intraläsionale Injektion einer SteroidKristallsupension, Kryotherapie oder Creme-PUVA etabliert.

Bei disseminierten Formen, großflächigem Befall und kosmetischer Beeinträchtigung besteht die Notwendigkeit einer systemischen Therapie. Dabei wurden Erfolge mit oralen Steroiden, Antimalariamitteln, Dapson, Balneofotochemotherapie beschrieben $[8,9]$. Eine weitere therapeutische Option stellt die Behandlung mit Retinoiden (Etretinat) mit oder ohne Kombination mit PUVA-Therapie dar [10].

Im Jahre 2001 berichten Schulze-Dirks und Petzoldt über das prompte Ansprechen einer Patientin mit Granuloma anulare disseminatum auf die Therapie mit FSE [6]. Die Ansammlung von CD4+-Lymphozyten in den Granulomen und die bei der Behandlung mit Fumarsäure auftretende Lymphopenie lagen der therapeutischen Überlegung zu Grunde [6]. Ein sehr guter Behandlungseffekt wurde hier bereits bei einer Dosierung von 3 Tabletten Fumaderm initial ${ }^{\circledR}$ gesehen. Weitere Berichte bestätigten den erfolgreichen Einsatz von FSE bei der Behandlung von insgesamt 8 Patienten mit disseminiertem Granuloma anulare und anderen granulomatösen Erkrankungen, z. B. der Sarkoidose oder Necro-
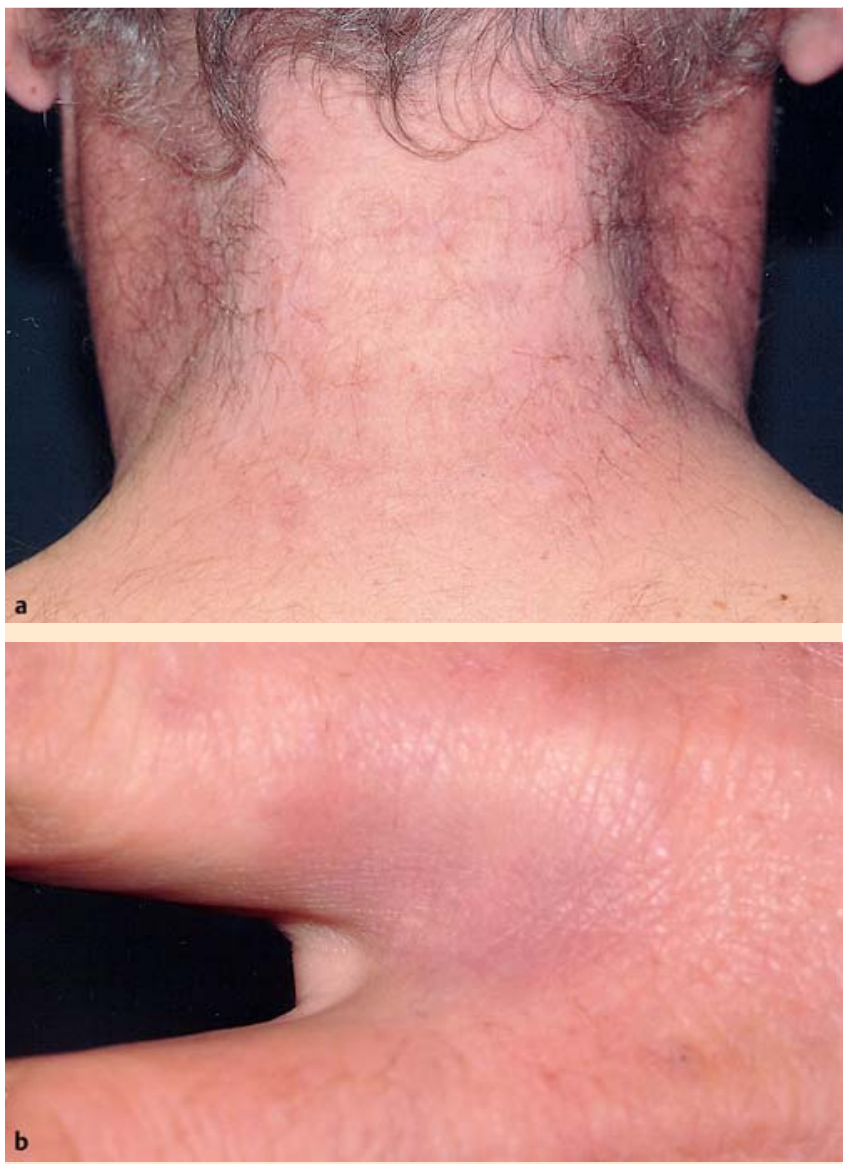

Abb. 4 Abgeheilter Hautbefund am Hals (a) und Handrücken (b) zwei Monate nach Therapieende.

biosis lipoidica, wobei Dosierungen von 1 bis 6 Tabletten Fumaderm ${ }^{\circledR}$ pro Tag nötig waren [11-13].

Fumaderm $^{\circledR}$ ist ein Gemisch aus Dimethylfumarat und den Kalzium-, Magnesium- und Zinksalzen ( $\bullet$ Tab. 1) des Monoethylesters Ethylhydrogenfumarat. Aktiver Wirkstoff des immunmodulatorisch wirkenden Medikamentes ist Dimethylfumarat. Fumaderm initial ${ }^{\circledR}$ und Fumaderm ${ }^{\circledR}$ sind in Deutschland seit 1995 für die Behandlung der Psoriasis zugelassen.

Der genaue Wirkmechanismus der FSE ist nicht endgültig geklärt [14]. Die bei vielen Patienten auftretende unerwünschte Wirkung der Lymphopenie wird als Hauptwirkung postuliert [14]. So wurde in Studien eine antiproliferative Wirkung auf T-Lymphozyten und eine daraus resultierende Normalisierung der gestörten TH1/TH2-Zytokinbalance bei der Psoriasis, außerdem eine Inhibition von Interferon gamma sowie eine Erhöhung der Interleukin-10-Sekretion nachgewiesen. Heute wird der antientzündliche Effekt durch Hemmung des nukleären Faktors kappa B, der an der Transkription von Genen, die für proinflammatorische Mediatoren wie Tumor-Nekrose-Faktor alpha und Interleukin 8 sowie für Ahdäsionsmoleküle wie E-Selektin und ICAM 1 kodieren, erklärt [14]. Allerdings beziehen sich die Arbeiten auf die Wirkung der FSE bei Psoriasis vulgaris. Da über die Ätiologie und Pathogenese des Granuloma anulare wenig bekannt ist, sind auch Angaben zum Wirkmechanismus der FSE bei dieser Erkrankung eher spekulativ.

Die wichtigsten Nebenwirkungen umfassen gastrointestinale Beschwerden wie Übelkeit, Bauchkrämpfe, Völlegefühl und Durchfälle, die bei etwa zwei Drittel der behandelten Patienten auftreten, aber auch Flush-Symptomatik und Müdigkeit. Die Nebenwir- 
Tab. 1 Zusammensetzung von Fumaderm initial ${ }^{\circledR} /$ Fumaderm $^{\circledR}$

\begin{tabular}{|c|c|c|}
\hline & $\begin{array}{l}\text { Fumaderm } \\
\text { initial }^{\circledR}\end{array}$ & $\begin{array}{l}\text { Fuma- } \\
\text { derm }^{\circledR}\end{array}$ \\
\hline Dimethylfumarate & $30 \mathrm{mg}$ & $120 \mathrm{mg}$ \\
\hline Ethylhydrogenfumarat Kalziumsalz & $67 \mathrm{mg}$ & $87 \mathrm{mg}$ \\
\hline Ethylhydrogenfumarat Mangnesiumsalz & $5 \mathrm{mg}$ & $5 \mathrm{mg}$ \\
\hline Ethylhydrogenfumarat Zinksalz & $3 \mathrm{mg}$ & $3 \mathrm{mg}$ \\
\hline
\end{tabular}

kungen sind dosisabhängig und treten typischerweise etwa ab der 4 Behandlungswoche auf. Bei unserem Patienten begannen Magenbeschwerden und Durchfälle ab der Dosierung von 3 Tabletten FSE pro Tag. Die Beschwerden nahmen erst bei weiterer Dosissteigerung von 5 Tabletten pro Tag an Intensität zu und waren für den Patienten gerade noch tolerabel.

Die geschilderten Symptome wurden von uns zunächst als unerwünschte Arzneimittelwirkungen der FSE gewertet. Jedoch war das zunehmende Unruhegefühl, Verstärkung der Durchfälle und eine rasche Gewichtsabnahme von $3 \mathrm{~kg}$ letztendlich wegweisend für die Diagnose einer Hyperthyreose. Im Gegensatz zu Differenzialblutbild, Leber- und Nierenwerten gehören die Schilddrüsenparameter nicht zu den empfohlenen Kontrollwerten im Rahmen der regelmäßigen Blutwertkontrollen. Eine Unterscheidung zwischen unerwünschter Wirkung der FSE-Therapie und einer Hyperthyreose kann dabei sehr schwierig sein. Es sollte deshalb bei der Symptomkonstellation von Durchfällen, Gewichtsabnahme, Unruhe und Tachykardie auch im Verlaufe der Behandlung eines Granuloma anulare an die Entwicklung einer Schilddrüsenerkrankung gedacht und entsprechende Laborwerte bestimmt werden. Ein Zusammenhang mit der FSE-Medikation wird nicht gesehen.

Mehrere Arbeiten erwähnen neben dem entgleisten Diabetes mellitus eine Schilddrüsenerkrankung als Realisationsfaktor für ein Granuloma anulare [1,5]. Möglicherweise war in unserem Fall die einlaufende Hyperthyreose ursächlich für das verzögerte Ansprechen auf die systemische Therapie. Bei unserem Patienten war ein Behandlungseffekt erst nach 12 Wochen bei 5 Tabletten Fumaderm ${ }^{\circledR}$ pro Tag zu verzeichnen, wogegen andere Autoren über gutes Ansprechen bereits nach 3 Wochen berichteten [6].

\section{Fazit}

\section{$\nabla$}

Neben den häufigen Verlaufsformen mit hoher Spontanheilungsrate gibt es beim Granuloma anulare auch seltene, sich progredient ausbreitende Formen.

Bei unserem Patienten bestand ein solches Granuloma anulare giganteum und erst die Behandlung mit FSE, nach unbefriedigendem Effekt von lokalen und intraläsionalen Steroiden, hat zu einer Abheilung der Hauteffloreszenzen geführt. Die bekannten unerwünschten Arzneimittelwirkungen können dabei gastrointestinale Symptome, Flush und Gewichtsabnahme umfassen. Da diese Symptome ebenfalls bei einer Hyperthyreose auftreten, muss bei zusätzlich auftretender Tachykardie und Unruhe des Patienten differenzialdiagnostisch auch an eine Hyperthyreose gedacht und eine Bestimmung entsprechender Laborparameter veranlasst werden.

Interessenkonflikte: Frau Dr. U. Proske gibt an, dass keine Interessenkonflikte bestehen.
Prof. G. Wozel ist Mitglied der Advisory Boards von Abbott GmbH \& Co KG, Astellas Pharma GmbH, Biogen Idec GmbH sowie Wyeth Pharma GmbH und hat Honorar für wissenschaftliche Präsentationen erhalten. Er ist in mehreren klinischen Studien als Principle Investigator eingebunden. Es liegt eine wissenschaftliche Kooperation mit Wyeth Pharma GmbH vor.

\section{Abstract}

\section{Granuloma annulare giganteum et disseminatum $\nabla$}

Granuloma annulare is a relatively common non-infectious disease with unknown aetiology sometimes associated with systemic conditions. It is characterized by a necrobiotic granulomatous inflammation. A rare manifestation of the disease is granuloma annulare giganteum exhibiting as hard plaques with lesions up to 20 centimetres. We report a 44 -year old male patient with a granuloma annulare giganteum et disseminatum. Despite topical therapy with glucocorticosteroids and repeated intralaesional steroid-injections further progression was observed. After beginning of treatment with fumaric acid ester (FAE) with the dosage recommended for treatment of psoriasis, a slow amelioration occured. Increasingly the patient reported sickness and diarrhea when the treatment reached four tablets of FAE per day. Finally a hyperthyroidism was confirmed with delay since the complaints initially were related to the treatment with FAE. As consequence by being aware of an increase of diarrhea, a fast loss of weight and tachycardia the development of a hyperthyroidism should be considered.

\section{Literatur:}

1 Kowalzick L. Granuloma anulare. Hautarzt 2005; 56: 1071 - 1083

2 Ryan J, Kastner D. Less common arthropathies. In: Klippel J, Stone J, Crofford L et al., Hrsg. Primer on the rheumatic diseases. 13. Aufl. New York: Springer; 2008: $501-502$

3 Mensing H, Jänner M. Granuloma anulare giganteum. Hautarzt 1981; 32: $256-257$

4 Pachinger W. Atypisches Granuloma anulare bei Diabetes mellitus mit iatrogenem Hyperinsulinismus. Wien Med Wochenschr 1991; 141: $47-48$

5 Gross $P$, Shelley $W$. The association of generalized granuloma annulare with antithyroid antibodies. Acta Derm Venereol 1971; 51: 59-62

6 Schulze-Dirks A, Petzoldt D. Granuloma anulare disseminatum - erfolgreiche Therapie mit Fumarsäureester. Hautarzt 2001; 52: 228 - 230

7 Buechner SA, Winkelmann RK, Banks PM. Identification of T-cell subpopulations in granuloma anulare. Arch Dermatol 1983; 119: 125 128

8 Goerdt S. Granulomatöse Erkrankungen. In: Braun-Falco O, Plewig G, Wolff $\mathrm{H}$ et al., Hrsg. Dermatologie und Venerologie. 5. Aufl. Heidelberg: Springer; 2005: 528-529

9 Wozel G. Innovative use of dapsone. Dermatol Clin 2010; 28: 599-610

10 Harth W, Richard G. Retinoide in der Therapie des Granuloma anulare disseminatum. Hautarzt 1993; 44: 693-698

11 Eberlein-König B, Mempel M, Stahlecker J et al. Disseminated granuloma annulare-treatment with fumaric acid esters. Dermatology 2005; 210 : 223-226

12 Breuer K, Gutzmer R, Völker B et al. Therapy of noninfectious granulomatous skin diseases with fumaric acid esters. Br J Dermatol 2005; 152: $1290-1295$

13 Gutzmer R, Kapp A, Werfel T. Erfolgreiche Therapie einer Haut- und Lungensarkoidose mit Fumarsäureestern. Hautarzt 2004; 55: 553 557

14 Mrowietz U, Rostami-Yazdi M, Neureither $M$ et al. 15 Jahre Fumaderm ${ }^{\circledR}$ : Fumarsäureester für die systemische Behandlung der mittelschweren und schweren Psoriasis vulgaris. JDDG 2009; 7 (Suppl 2): 1-16 